\title{
Evidence-Based Psychotherapy: Advantages and Challenges
}

\author{
Sarah C. Cook $^{1} \cdot$ Ann C. Schwartz ${ }^{1}$ Nadine J. Kaslow ${ }^{1}$
}

Published online: 26 June 2017

(C) The American Society for Experimental NeuroTherapeutics, Inc. 2017

\begin{abstract}
Evidence-based psychotherapies have been shown to be efficacious and cost-effective for a wide range of psychiatric conditions. Psychiatric disorders are prevalent worldwide and associated with high rates of disease burden, as well as elevated rates of co-occurrence with medical disorders, which has led to an increased focus on the need for evidence-based psychotherapies. This chapter focuses on the current state of evidence-based psychotherapy. The strengths and challenges of evidence-based psychotherapy are discussed, as well as misperceptions regarding the approach that may discourage and limit its use. In addition, we review various factors associated with the optimal implementation and application of evidence-based psychotherapies. Lastly, suggestions are provided on ways to advance the evidencebased psychotherapy movement to become truly integrated into practice.
\end{abstract}

Keywords Evidence-based psychotherapy · Evidence-based treatment $\cdot$ Evidence-based treatment strengths .

Evidence-based treatment challenges $\cdot$ Evidence-based treatment misperceptions $\cdot$ Evidence-based therapy

\section{Background}

Psychiatric disorders are prevalent worldwide [1] and are associated with high rates of disease burden, including elevated rates of morbidity and mortality [2,3]. In addition, there is a high rate of co-occurrence between psychiatric and medical

Nadine J. Kaslow

nkaslow@emory.edu

1 Emory University School of Medicine, Atlanta, GA, USA disorders $[4,5]$. When psychiatric disorders co-occur with medical problems, not only are the medical symptoms more problematic, but the treatment of the medical condition is often more complicated [6]. For example, there is often lowered levels of treatment adherence and higher levels of healthcare service utilization, with its associated costs [4]. Therefore, increasing attention has been paid to the need for evidencebased pharmacological and psychotherapeutic interventions for a range of psychiatric disorders $[7,8]$.

This chapter focuses on the current state of evidence-based psychotherapy. These psychotherapies are efficacious, beneficial, and cost-effective for myriad psychiatric disorders $[9$, 10]. Moreover, people prefer psychotherapy to pharmacological treatments [11]. Unfortunately, despite the sizable evidence base, there is a significant gap between the availability of effective psychotherapies and the delivery of such interventions in the community [12].

\section{History of Evidence-Based Practice}

The roots of evidence-based medicine go back centuries [13]. Yet evidence-based practice (EBP; i.e., evidence-based treatment) did not became a "hot topic" in medicine until the 1990 s, as attention began to be paid to the value of using evidence-based medicine to support decision-making in practice, educational, and policy contexts. This laid the groundwork for the adoption of EBP in medicine, as well as other healthcare professions.

The term "evidence-based" was first used by Eddy in 1987 in his workshops on designing clinical practice guidelines in medicine. In the 1990s, the phrase began to be used in relation to a clinical decision-making approach informed by published findings [13-15]. The term was first formally defined by Sackett, often viewed as the father of this movement, and his 
colleagues in 1996. They stated, evidence-based medicine is the "conscientious, explicit, and judicious use of current best evidence in making decisions about the care of individual patients" [16]. They noted that it requires the integration of the practitioner's clinical expertise with the best available data gleaned from systematic investigations [16]. Over time, the concept has expanded and now includes consideration of patients' preferences, actions, clinical state, and circumstances [17]. The key steps of EBP in medicine include formulating the clinical question based on the presenting problem, critically evaluating the pertinent literature with regard to its validity and usefulness for a given patient, implementing the research findings in clinical practice, and evaluating the outcomes [18].

\section{EBP and Psychotherapy}

The American Psychological Association developed a policy on the EBP of psychotherapy [19] that follows the definition put forth by Sackett et al. [16] and the Institute of Medicine [20]. This policy emphasizes integrating the best-available research with clinical expertise in the context of the patient's culture, individual characteristics, and personal preferences. The best research evidence refers to data from meta-analyses, randomized controlled trials, effectiveness studies, and process studies, as well as information obtained from single-case reports, systematic case studies, qualitative and ethnographic research, and clinical observation. The applicability of evidence to specific cases must be considered, which ties to the emphasis placed on integrating clinical expertise and specific clinical information vis-à-vis the patient with the pertinent research evidence to make clinical decisions, implement treatment plans, foster a therapeutic alliance, and achieve positive outcomes. This policy makes clear that the effectiveness of any psychotherapy is influenced by the unique characteristics of each patient, such as developmental history and life stage, personal problems, strengths, personality structure, functional status, readiness to change or engage in psychotherapy, degree of social support, and family and sociocultural factors. The policy also highlights consideration of the patient's environment when choosing an evidence-based psychotherapy modality and notes the role of healthcare disparities and specific stressors (e.g., unemployment, major life events).

One key goal of EBP psychotherapy is to maximize patient choice about options. Since the outset of EBP generally and the psychotherapy movement specifically, patients' preferences are prioritized, which affords them active choices. Clinical decisions associated with evidence-based psychotherapies optimally are made collaboratively with the patient, based on the best available evidence, with attention to costs, benefits, available resources, and options [21, 22]. Such decision-making involves ongoing monitoring and adjustment.

\section{Strengths of Evidence-Based Psychotherapy}

There are advantages of evidence-based psychotherapies for practitioners, clinical teams, and patients [18]. It has been argued that for practice to be ethical it is imperative that it is guided by the relevant data [23]. By incorporating research into clinical practice, providers use research-driven evidence rather than rely solely on personal opinion. Using empirical evidence reduces opinion-based bias of recalling only "successes". When practiced appropriately, EBP can complement clinical expertise when making judgments. Incorporating research inevitably promotes the development of guidelines, databases, and other clinical tools that can help clinicians make critical treatment decisions, particularly in community-based settings [24]. Evidence-based psychotherapy encompasses both scientific and local evidence, such as diagnostic patient information, situational information including cost and time constraints, and the provider's judgment and experience to achieve the best outcome [25].

Applying evidence-based principles ensures that providers use the best existing evidence as a starting framework, while simultaneously affording them flexibility to individualize treatment. More specifically, evidence-based practice ensures that providers critically assess the data available and apply it to individual patient circumstances. When the evidence is appraised and fully understood, providers can decide if and how to incorporate it into practice. In addition, using evidence-based psychotherapies helps providers determine treatment plans, including in situations in which there are limited data or experience [26]. In fact, in patients with multiple medical and psychiatric comorbidities, using evidence-based treatments offers providers a starting point to develop complex treatment plans [27].

One misperception of evidence-based psychotherapy use is that in order to be useful, the evidence must be from a randomized controlled trial, which is typically challenging for many fields but particularly for psychotherapies. In fact, the evidence supporting the wide variety of psychotherapies available can include numerous methodologies as long as the evidence is assessed and applied appropriately in clinical decision-making [25]. Ideally, practitioners who actively employ EBPs save time, money, and resources by avoiding treatments with little or questionable effectiveness for their patients.

Training professionals to utilize EBPs enhances people's knowledge, skills, and attitudes and clinical acumen [28, 29], especially when accompanied by a focus on reflection [30]. In addition, training in the use of myriad evidencebased psychotherapies ensures that providers are familiar with the state-of-the-field and have depth and diversity in their clinical practice. Training in an EBP, coupled with an active and ongoing learning process, is required for professionals to facilitate patient change and other positive outcomes [28, 31]. 
Ultimately, the goal of EBP is the promotion and implementation of psychotherapies that are safe, consistent, and cost-effective [32]. As a result, evidence-based psychotherapies are associated with higher quality and more accountability [29], as well as the enhancement of the health and wellbeing of the public [19]. Because providers adhere less to evidence-based methods over time, quality of care diminishes with increasing years of experience. In addition, providers with more experience may be less up to date with current knowledge, guidelines, or standards of care, and, as a result, their patients may have poorer treatment outcomes [33].

\section{Challenges of Evidence-Based Psychotherapy}

Despite the many strengths of using evidence-based psychotherapy, there are challenges that must be considered [30, 34]. First, concerns have been raised about the generalizability of the findings, given that the conditions and characteristics of randomized controlled treatment outcome research versus those of real-world clinical practice differ significantly [34]. For example, research samples often under-represent minority populations or patients with comorbid conditions [35] and, as a result, evidence-based psychotherapies often are not effective for individuals with complex multimorbidities or from sociodemographic groups for which the intervention has yet to be tested [30]. In addition, many psychotherapy trials for depression and anxiety recruit participants with limited psychosocial stressors given their confounding nature. However, in actual practice, most patients face these stressors and it is unclear how well the purported evidence-based psychotherapies will treat these individuals [34]. Evidence-based psychotherapy is also challenging to apply to individuals given that the evidence is based on a composite of multiple subjects, with limited attention to the impact of individual factors and influences on the patient's health.

Second, there are a number of marked differences between the processes of commonly practiced psychotherapies and EBP. For example, EBP tends to focus on ameliorating symptoms or disorders, whereas many people seek out psychotherapy to cope more effectively with life's challenges and have a greater sense of meaning in their lives. In addition, psychotherapies typically prioritize empowerment and supporting people in achieving their own treatment goals, whereas evidence-based approaches risk not attending to patients as agents of change or self-healers. As another example, diverse forms of psychotherapy guided by myriad theoretical perspectives or a combination of such models are practiced. The majority of evidence-based psychotherapies are cognitive-behavioral, whereas many practitioners employ existential-humanistic, interpersonal, psychodynamic, systemic, and/or integrative models [9]. While there appears to be a divide between evidence-based research and practice and clinical application, evidence-based psychotherapies synthesize new knowledge when providers test evidence-based guidelines and adapt them to cohort specific circumstances.

Third, reliance on scientific research is problematic, as for many practices the level of evidence required to be considered "evidence-based" is lacking or unattainable. There are no agreed-upon criteria for determining if a psychotherapy is evidence-based or empirically supported and what is statistically significant and suggestive of empirical support may not be clinically relevant [12, 30, 34]. In addition, often times the randomized controlled trials compare an active intervention with a waitlist control or attention control condition that does not exist in the community. Until efficacy and effectiveness studies include treatment conditions that resemble practice in the real world, it is challenging to draw conclusions from the existing data that can meaningfully affect clinical practice [36]. Similarly, there are limited data regarding the mechanisms of change in an intervention that produce effective outcomes [37].

Professionals must have timely access to information for optimal implementation. This is challenging in that there are often lags between conduction of research and publication, and then from publication to adoption into practice or policy. As with any research modality, evidence-based psychotherapies are subject to biases, such as sponsorship of research, methodologies used, subjects chosen, and publications, which may impact on the credibility of the particular treatment.

Fourth, overemphasis on using evidence-based psychotherapies could erroneously ignore other clinical tools, most notably professionals' own clinical experience. Similarly, when evidence-based psychotherapies are applied too rigidly, there is risk of diminishing their value, particularly if applied to patients for whom effectiveness will be limited, causing the psychotherapy (and policy) to be called into question. Such over-reliance on rules may result in psychotherapeutic practice that is management driven, rather than patient-centered [30].

Finally, from the practitioner's standpoint, dedicated use of evidence-based psychotherapies could impose burden in terms of continuing education and training. Clinicians using evidence-based psychotherapies must maintain up-to-date knowledge of the latest evidence supporting current or new methods, which, of course, takes a considerable amount of time. Providers must have adequate training to identify and implement the most appropriate psychotherapy for a patient [38]. Other components, such as database and journal access, in addition to training, can be costly and challenging to locate for more remote clinicians.

\section{Misperceptions of Evidence-Based Psychotherapy}

Resistance to using evidence-based psychotherapies results from clinicians and patients, often due to misunderstandings or 
misperceptions of the role of evidence-based psychotherapies [23]. Exposure to caricatured versions of evidence-based psychotherapies causes oversimplification of treatments that could easily discourage clinicians from utilizing them [39]. There is a misperception that evidence-based psychotherapies are merely "cookbook" practice instructions that force clinical professionals to replace their judgment with "manualized" procedures. In reality, most guidelines, including those of the American Psychological Association [19], strongly recommend incorporation of clinical expertise and judgment into applicable practice of evidence-based psychotherapies. Evidence-based psychotherapies are more appropriately considered as a "map" of potential routes, with the practitioner determining which treatment path to take based on the unique history and presentation of the patient. Even empirically supported psychotherapies do not generally recommend predetermined responses to patients' behaviors or rigid adherence to protocols [40]. Inflexibility in treatment protocols can lead to undesirable treatment outcomes [41]. As a result, Kendall et al. [42] advocate for a model that embodies "flexibility within fidelity" in which practitioners follow basic treatment guidelines of an evidence-based psychotherapies without rigid adherence. Such fidelity should relate to core components, rather than specific techniques, given that it is fidelity to core components during treatment that ensures good outcomes [43].

It is often assumed that using an evidence-based psychotherapy means that patients' values and preferences are ignored in order to pursue a prescribed, rigid treatment plan. This plan is often viewed as a costingcutting measure rather than a first-line modality. However, appropriate use of evidence-based psychotherapy incorporates clinical expertise, which, by definition, requires incorporating patient values, preferences, and individual circumstances as integral parts of decisionmaking. Incorporating evidence-based psychotherapy and clinical expertise creates the most effective means of treatment resulting in cost savings.

Many clinicians hold a misperceived idea that the psychotherapy provided could never meet EBP criteria or standards, because data are insufficient or flawed. While all research has limitations, the key to evidencebased psychotherapies is to use the best available evidence and differentiate between limitations and "fatal" flaws. Treatments without sufficient evidence may be used with caution and careful monitoring and in accord with clinical expertise and patient preference.

\section{Implementation and Application of Evidence-Based Psychotherapy}

In this section, attention is paid to various factors association with the optimal implementation of evidence-based psychotherapies. The factors addressed include relationships, fidelity, flexibility context, and providers.

\section{Relationships}

While much attention has been paid to the value of evidencebased psychotherapies, there is considerable evidence that the therapeutic relationship makes substantial and consistent contributions to psychotherapy outcomes independent of the type of treatment [44-46]. The relationship acts in concert with treatment methods, patient characteristics, and practitioner qualities in determining effectiveness. In fact, the therapeutic relationship accounts for why patients improve, or fail to improve, at least as much as the particular treatment method. In addition, adapting or tailoring the therapeutic relationship to specific patient characteristics, including diagnoses, further enhances the effectiveness of treatment [44]. As a result, any discussion of evidence-based psychotherapies must include attention to evidence-based relationships.

Demonstrably effective elements of the relationship include forming a positive therapeutic alliance in individual, youth and family psychotherapy; cohesion among patients in a group therapy setting; empathy; and eliciting patient feedback [44]. Elements that are probably effective include goal consensus, collaboration, and positive regard and support [44]. There is insufficient, yet promising, research on the elements of congruence or genuineness, repairing alliance ruptures, and managing countertransference. Ineffective elements of the therapeutic relationship can curtail progress or contribute to negative outcomes [44]. Ineffective elements include inappropriate or ill-timed confrontations, negative processes, or making assumptions about the patient. Therapist centricity, or providing treatment that revolves around the psychotherapist's goals or agenda, is also ineffective and impedes progress. Similarly, rigidly adhering to a uniform procrustean bed of psychotherapy for all patients ineffectively binds the individual to ineffective treatment [44].

Efforts to promulgate evidence-based psychotherapy must include a focus on the therapeutic relationship. There are several recommendations to ensure the therapeutic relationship makes evidence-based psychotherapy as effective as possible. First, a comprehensive understanding of effective (and ineffective) psychotherapy must consider how the therapeutic relationship acts in concert with other determinants and their optimal combinations. Practice and treatment guidelines should explicitly address therapy behaviors and qualities that promote a facilitative therapeutic relationship. Psychotherapists must prioritize understanding their patients, recognizing them as agents of change within sessions, supporting them as self-healers, and intentionally shaping their interventions based on being attuned to the patients' experiences of psychotherapy. This involves viewing psychotherapy as a process of change through structured curiosity 
and deep engagement in pattern identification and narrative reconstruction. Psychotherapists must be caring, understanding, and accepting, which allows patients to internalize positive messages and enter the change process of developing selfawareness [47]. In addition, they must recognize that professional structures create credibility and clarity, but cast suspicion on care within the relationship. Psychotherapists who forge productive relationships with their patients appreciate that psychotherapy progresses as a collaborative effort with discussion of differences between both parties. They also recognize that patients' agency with regard to decision-making and the therapeutic process increases the likelihood that responsive interventions are employed that fit their needs and that result in positive outcomes.

\section{Fidelity}

In addition to attention to the therapeutic relationship, evidence-based psychotherapies that yield good outcomes are those that are practiced with a high level of fidelity such that the core components of the psychotherapy are implemented [43]. The core components refer to the basic elements of the evidence-based psychotherapy that are required for applicability and validity of the intervention [48]. Core components are often defined by the evidence-based psychotherapy developers or in policy guidelines and help describe population characteristics, content of the psychotherapy, context or setting of the intervention, and sequence of the treatment. For example, population characteristics could include adult women with post-traumatic stress disorder; the content is described as 5 lessons on 5 themes of emotion regulation; the context is in a clinic group therapy room on a weekly basis; and the sequence is described as first, emotional identification, then promotion of positive emotions.

Fidelity to an evidence-based psychotherapy is important because when elements of the treatment are changed, the practice is no longer the same as the researched practice. In other words, psychotherapists are no longer implementing an EBP when it no longer resembles the practice in the evidence. Consistency, achieved through fidelity, allows for stronger statements about the efficacy of a practice. Evidence-based psychotherapies implemented with fidelity are more likely to achieve the desired outcomes (as described in the evidence). Many tools for maintaining fidelity are available and include toolkits and training manuals, ongoing training and supervision, and fidelity monitoring and fidelity scales.

Fidelity adherence when using evidence-based psychotherapies faces some challenges. Unfortunately, other than in efficacy research, it is not generally feasible to closely monitor fidelity in real-world implementation. Rigid application of fidelity reduces the usability of a particular psychotherapy. For example, labeling a treatment as only for depression without anxiety eliminates application to many patients with depression. Maintaining fidelity often requires time and resources for training and ongoing monitoring. In fact, programs with high staff turnover requiring repeated trainings of newhires, leadership or government requirements, and extensive training for the psychotherapy can influence fidelity over time, particularly for larger-scale implementation of evidence-based psychotherapies [49]. Some evidence-based psychotherapies are simply more challenging to implement with fidelity than others. Fidelity requires conscientious application of the principles of the evidence-based psychotherapy to practice, which is subject to problems in translation or competence, particularly in the context of dissemination efforts.

\section{Flexibility}

While fidelity is a crucial component of successful evidencebased psychotherapy practice, implementation with flexibility is also necessary. Flexibility refers to areas where the application of the psychotherapy differs from the specific EBP, such as deviations from manual-based protocol or individualized applications based on patient characteristics. Flexible implementation should still retain core components of the evidencebased psychotherapy. Flexibility may be desirable in a number of different situations. For example, therapists may use flexibility to build rapport, select treatment modality, or alter the pacing of the intervention in order to assist a patient who has difficulty learning multiple skills rapidly or integrating particular aspects of the treatment. Flexibility also may be warranted in working with people's reactions to current life stressors, such as a death in the family or other traumatic events, as these often fall outside of the evidence-based protocol. Moreover, flexibility may be necessary in situations in which individuals present with comorbid conditions, as these must be taken into account in treatment selection and implementation. For example, patients struggling with obsessive thoughts in addition to post-traumatic stress disorder will likely need an alternative therapy prior to starting evidence-based trauma exposure treatment. Modality changes may also be needed for unique patient situations, such as telephone or internet sessions if the patient travels frequently. In addition, some patients prefer the use of technology (e.g., smartphones and other mobile devices) for the receipt of psychological treatments and this may require flexible modification of an EBP traditionally administered in the more usual office setting [8]. Finally, many patients benefit from booster sessions or skills refreshers that are not necessarily built into evidence-based protocols and therapists must be open to accommodating such needs.

Incorporating flexibility into an evidence-based psychotherapy treatment is not without challenges. Flexibility can be difficult to include in research on evidence-based psychotherapy as the variation from the protocol becomes challenging to monitor and introduces confounding factors. Overuse of flexibility reduces fidelity, which as discussed earlier, is 
critical to maintaining an effective evidence-based psychotherapy. The challenge psychotherapists face is walking the fine line between flexible implementation of an evidencebased psychotherapy and the maintenance of the core components of the intervention. It is for this reason therefore that evidence-based psychotherapies are increasingly being developed that provide guidance with regard to both flexibility and fidelity. For example, the Skills Training in Affective and Interpersonal Regulation-Narrative Therapy (STAIR-NT) protocol includes a wide range of sessions per topic, allows for nonprotocol sessions to address individual patient crises, and encourages optional booster sessions [50].

\section{Context}

Effective implementation must also take context into account. In terms of context, evidence-based psychotherapies are implemented across a multitude of settings, including private practices, Veterans Health Administration facilities, counseling centers, medical centers, and educational systems to name a few. While some evidence-based psychotherapies are designed for specific contexts, others are formulated for implementation across multiple contexts. As with other components of evidence-based psychotherapies, the context of development should be considered when selecting a treatment.

Although the intent is generally to implement evidencebased psychotherapies across multiple settings, therapists should consider the extent of applicability to their patient population. For example, the Veterans Health Administration (VHA) often uses guidelines for various disorders and postdeployment health, and evidence-based psychotherapies are often a major cornerstone of treatment. Indeed, the VHA has been a leader in training staff in the delivery of evidencebased interventions and in disseminating and implementing these psychotherapies [51]. Data from program evaluations reveal that such training has resulted in positive outcomes for psychotherapies, such as greater clinical competence and self-efficacy [51]. In addition, patient outcomes have been encouraging in response to these evidence-based psychotherapies as well, in terms of both symptom reduction and improvements in quality of life. The large-scale dissemination efforts in this context suggest that it is feasible to overcome the science to practice based gap that has existed historically vis-à-vis evidence-based psychotherapy practice and that efforts to bridge this gap can yield positive outcomes [51].

Unfortunately, this has led to concern that treatment at the VA is based on modules and algorithms with limited flexibility or individualized care and may not be suited to all settings. For example, medical settings face challenges with the stepped care or algorithm-based care rather than treatment tailored to the individual needs of the patient. In the medical context, motivational interviewing is an effective modality for addressing behavioral health issues, such as substance use disorders, obesity, chronic pain, and diabetes, and is a valuable evidence-based psychotherapeutic intervention for depression that is flexible in its delivery and easily integrated into primary care settings [52, 53].

The education system is a very different type of site that provides evidence-based psychotherapies to people diagnosed with autism spectrum disorders, depression, and anxiety. In addition, the education system provides suicide prevention screening for all students. Those in educational settings face numerous challenges to the implementation of EBP, including the cost of manuals for evidence-based interventions, selection of a specific EBP intervention, provider reluctance to use $\mathrm{EPB}$, and stigma regarding the interventions from students, parents, and teachers [54].

Although evidence-based psychotherapies are typically thought of as primarily oriented toward cognitive-based therapies (CBT), there are evidence-based psychotherapies associated with a multitude of therapeutic orientations. When selecting evidence-based psychotherapies, it is important to consider the therapeutic orientation in terms of what is most applicable to the patient and what is most authentic to the provider. Examples of first- and second-wave CBTs include applied behavioral analysis, behavioral therapy for various disorders, behavioral parenting training, CBT for various disorders (depression, anxiety, psychosis, etc.), cognitive processing therapy, exposure therapy and prolonged exposure therapy, lifestyle modification, and behavior couples and family therapy. Third-wave CBT is a modality that is sensitive to context and functions of psychological phenomena and focuses on metacognition, cognitive fusion, emotions, acceptance, mindfulness, dialectics, spirituality, and the therapeutic relationship. Examples of third-wave CBT include behavioral activation, schema therapy, acceptance and commitment therapy, cognitive behavioral analysis system of psychotherapy, dialectical behavior therapy, metacognitive therapy, mindfulness-based cognitive therapy, and mindfulness-based stress reduction. Other orientations of evidence-based psychotherapies include interpersonal, emotion focused, systemic (e.g., functional family therapy, multisystemic therapy), psychodynamic, and integrative models including the STAIR-NT, parent-child interaction therapy, trauma-based CBT, and integrative behavioral couples therapy. As may be evident from these listings, there are evidence-based therapies for young people [55], adults, older adults, and couples and families [12, 55-57].

Most evidence-based psychotherapies are designed for single-diagnosis conditions, while the reality is that many patients have multiple comorbid conditions that all require treatment [58]. Fortunately, some evidence-based psychotherapies are specifically designed for comorbid conditions or have research available for comorbid conditions. For example, the Seeking Safety protocol addresses post-traumatic stress disorder and comorbid substance use disorder [59]. Adolescent 
Coping with Depression [60] treats young people with both depression and conduct disorder. Motivational interviewing, CBT, and/or family/caregiver interventions can address comorbid substance use disorders and mood and/or schizophrenia spectrum disorders. Recently there has been movement toward a transdiagnostic approach for addressing comorbid disorders effectively with evidence-based psychotherapies.

\section{Provider}

A crucial component of evidence-based psychotherapy is the provider. Many evidence-based psychotherapies imply that psychologists are the primary providers. However, given the multitude of contexts and settings using evidence-based psychotherapies, there is an equally wide variety of providers, including physicians, nurses, social workers, professional counselors, and graduate students. Therapist variables must be considered, including individual attributes such as training, clinical experience, theoretical orientation, and therapist attitudes towards EBP [61, 62].

An essential part of most evidence-based psychotherapies is training, including both initial training and ongoing training and supervision. Complex interventions may require additional provider training and skill. Therapist knowledge improves and attitudinal change occurs following training, and the method for training (particularly ongoing) influences ease of implementation, accessibility, and desirability. Organizational variables and culture influence training and consequent therapist uptake and adoption of evidence-based psychotherapy [63]. Training can occur during a specific period or be part of life-long learning, and typically includes didactics, manual review, practice, and supervised experience, often in groups and with review of actual case materials, as well as training to become a trainer. The training method is an important vehicle and active learning, an interactive process that uses action and reflection has been an effective teaching strategy [28, 31].

Clinical experience is an additional important provider variable and the therapists in EBP will vary with some being more skilled. Research settings often rely on trainees in various disciplines and specialties; however, therapists in clinical trials are selected for their expertise and may be removed from the study if they cannot deliver the treatment skillfully [61]. In clinical settings, there often is a combination of providers with different specialties and levels of training that can create challenges, as there are no clear replicable procedures for how to tailor EBP to an individual patient, and different providers may not reliably select a similar individualized plan when presented the same case [34].

Provider theoretical orientation and attitudes towards EBP are key factors. Provider training and level of professional development should be considered as those who trained using evidence-based assessment protocols are more likely utilize these methods. Moreover, therapists who have an allegiance to other treatments may bias the outcomes and also have issues with adherence to the treatment (fidelity concerns as above). In addition, provider attitudes are influential in the willingness to adopt and implement EBP and educational attainment is positively associated with endorsement of EBP and attitudes toward its adoption [61, 62].

\section{Future Directions}

The EBP and associated evidence-based psychotherapy movements have countless advantages. The dissemination and implementation of evidence-based psychotherapies can promote recovery of individuals who present with a myriad of psychiatric disorders [51]. Despite the availability of effective evidence-based interventions for a range of common mental health disorders [10], consistent provision of such interventions is not widespread [64]. For efforts to be more successful in ensuring that evidence-based psychotherapies are practiced more consistently by practitioners in multiple settings, the efficacy and effectiveness treatment research literature must be bolstered, the mechanisms of change associated with intervention effectiveness must be articulated, clinical guidelines that integrate information on mechanisms of change must be developed and disseminated, measures of intervention quality must be developed and utilized, and systematic methods for evidence-based intervention implementation and ongoing utilization that include training practitioners in these approaches must be created [12]. Systems that have been effective in their dissemination and implementation activities, such as the VHA, can serve as models for other service delivery settings [51]. More wide-scale efforts will be optimized if they are guided by the research on dissemination science.

In addition, for the EBP movement in general and the evidence-based psychotherapy movement more specifically to advance and become truly integrated into practice [65], many of the unintended consequences of this movement need to be acknowledged and addressed [30]. More specifically, there needs to be greater appreciation of the value of individual practitioners being flexible in their implementation of protocols based on the unique needs and preferences of their individual patients [30], along with their own clinical expertise [49]. In a related vein, the approach must be practiced in a manner that places greater value on patient-centered care and the relationship between the patient and the healthcare provider [30]. To this end, there may need to be greater prioritization given to the evidence-based relationships movement [44]. There also needs to be greater attention paid to the factors common across psychotherapeutic approaches and the guiding principles of therapeutic change and processes, rather than to specific empirically supported treatments or even treatment guidelines $[61,66]$. 
Training in evidence-based psychotherapy must move beyond teaching people specific manualized treatments and emphasizing a high level of fidelity to such intervention practices toward an emphasis on applying such practices to real-world situations [30]. For example, this would require more attention to ensuring the cultural relevance of the delivery of evidencebased interventions [35, 67].

For the evidence-based psychotherapy movement to continue to advance and to inform clinical practice, a number of steps must be taken with regard to the research efforts [8, 14, 68]. The relevance of various sociodemographic factors (e.g., age, race/ethnicity, sex, disability) in relation to intervention efficacy, adoption, and impact must be ascertained. The conditions under which various psychotherapies are effective versus ineffective must be determined. More attention needs to be paid to the mechanisms of change that account for intervention outcomes. Moreover, there needs to be more attention paid to developing and evaluating interventions that combine psychotherapeutic and pharmacological interventions, given the value of each for various psychiatric disorders. Providers must be given opportunities for continuing education in evidence-based psychotherapies to help ameliorate the inverse relationship of experience and EBP use. The known costeffectiveness of using EBP would more than offset the cost of provider training. Interventions found to have good empirical support under controlled conditions need to be evaluated more thoroughly in real-world settings. Implementation issues also need to be attended to earlier in the research process. Finally, the research agenda needs to be creative and imaginative [30]. This can be accomplished by greater incorporation of interprofessional cadres of researchers, inclusion of qualitative, as well as quantitative, methods, and involvement of participants who can potentially benefit from the psychotherapies being created and tested. Clinicians and researchers must collaborate to achieve shared goals of achieving measurable outcomes and improving patient well-being.

Required Author Forms Disclosure forms provided by the authors are available with the online version of this article.

\section{References}

1. Steel, Z., Marnane, C., Iranpour, C., et al., The global prevalence of common mental disorders: A systematic review and meta-analysis 1980-2013. Int J Epidemiol 2014. 43: p. 476-493.

2. Whiteford, H.A., L. Degenhardt, J. Rehm, et al., Global burden of disease attributable to mental and substance use disorders: findings from the Global Burden of Disease Study 2010. Lancet, 2013. 382: p. $1575-1586$.

3. Walker, E.R., R.E. McGee, and B.G. Druss, Mortality in mental disorders and global disease burden implications: a systematic review and meta-analysis. JAMA Psychiatry 2015. 72: p. 334-341.

4. Lewis, V.A., C.H. Colla, K.T. Tierney, et al., Few ACOs pursue innovative models that integrate care for mental illness and substance abuse with primary care. Health Affairs, 2014. 33: p. 1808-1816.

5. Goodell, S., B.G. Druss, and E.R. Walker, Mental disorders and medical comorbidity (Policy Brief No. 21). 2011, The Synthesis Project: The Robert Wood Johnson Foundation: Princeton, NJ.

6. Kaslow, N.J., S. Kapoor, S. Dunn, et al., Psychologists' contributions to patient-centered medical homes. J Clin Psychol Med Set 2015. 22: p. 199-212.

7. Huhn, M., M. Tardy, L.M. Spineli, et al., Efficacy of pharmacotherapy and psychotherapy for adult psychiatric disorders: a systematic overview of meta-analyses. JAMA Psychiatry, 2014. 71: p. 706-715.

8. Gaudiano, B.A. and I.W. Miller, The evidence-based practice of psychotherapy: facing the challenges that lie ahead. Clin Psychol Rev 2013. 33: p. 813-824.

9. Emmelkamp, P.M., D. David, T. Beckers, et al., Advancing psychotherapy and evidence-based psychological interventions. Int $\mathrm{J}$ Methods Psychiatric Res 2014. 23 (Suppl. 1): p. 58-91.

10. Castelnuovo, G., G. Pietrabissa, R. Cattivelli, et al., Not only clinical efficacy in psychological treatments: clinical psychology must promot cost-benefit, cost-effectiveness, and cost-utility analysis. Front Psychol 2016. 7: p. 563.

11. McHugh, R.K., S.W. Whitton, A.D. Peckham, et al., Patient preference for psychological vs pharmacologic treatment of psychiatric disorders: a meta-analytic review. J Clin Psychiatry, 2013. 74: p. 595-602.

12. Institute of Medicine, ed. Psychosocial interventions for mental and substance use disorders: a framework for establishing evidencebased standards. 2015, The National Academies Press: Washington DC.

13. Claridge, J.A. and T.C. Fabian, History and development of evidence-based medicine. World J Surg 2005. 29: p. 547-553.

14. Smith, R.D. and D. Rennie, Evidence-based medicine - an oral history. JAMA 2014. 311: p. 365-367.

15. Evidence-Based Medicine Working Group, Evidence-based medicine: a new approach to teaching the practice of medicine. JAMA 1992. 268: p. 2420-2425.

16. Sackett, D.L., W.M.C. Rosenberg, J.A.M. Gray, et al., Evidence based medicine: what it is and what it isn't. BMJ, 1996. 312: p. 71-72.

17. Haynes, R.B., P.J. Devereaux, and G.H. Guyatt, Clinical expertise in the era of evidence-based medicine and patient choice. ACP J Club 2002. 136: p. A11-4.

18. Rosenberg, W.C. and A. Donald, Evidence based medicine: an approach to clinical problem-solving. BMJ 1995. 310: p. 1122-1126.

19. American Psychological Association, Evidence-based practice in psychology: APA Presidential Task Force on Evidence-Based Practice in Psychology. Am Psychol 2006. 61: p. 271-285.

20. Institute of Medicine, Crossing the quality chasm: a new health system for the 21 st century. 2001, Washington D.C.: National Academy Press.

21. Edwards, A. and G. Elwyn, Shared decision-making in health care: achieving evidence-based patient choice. 2009, New York NY: Oxford University Press.

22. Craighead, W.E. and B.W. Dunlop, Combination psychotherapy and antidepressant medication treatment for depression: for whom, when, and how. Annu Rev Psychol 2014. 65: p. 267-300.

23. Blease, C.R., S.O. Lilienfeld, and J.M. Kelley, Evidence-based practice and psychological treatments: the imperatives of informed consent. Front Psychol 2016;7:1170.

24. Nelson, T.D., R.G. Steele, and J.A. Mize, Practitioner attitudes towards evidence-based practice: themes and challenges. Adm Policy Ment Health 2006. 33: p. 398-409.

25. Rousseau, D.M. and B.C. Gunia, Evidence-based practice: the psychology of EBP implementation. Annu Rev Psychol 2016. 67: p. 667-692. 
26. Barends, E.G.R. and R.B. Briner, Teaching evidence-based practice: lessons from the pioneers. An interview with Amanda Burls and Gordon Guyatt. Acad Manag Learn Educ 2014. 13: p. 476-483.

27. Roberts, A.R. and K.R. Yeager, Evidence-based practice manual. 2004, New York, New York: Oxford University Press.

28. Beidas, R.S. and P.C. Kendall, Training in evidence-based practice: a review. Clin Psychol (New York) 2010. 17: p. 1-30.

29. Spring, B., Evidence-based practice in clinical psychology: what it is, why it matters; what you need to know. J Clin Psychol 2007. 63: p. 611-631.

30. Greenhalgh, T., J. Howick, and N. Maskrey, Evidence based medicine: a movement in crisis? BMJ 2014. 348:g3725.

31. Beidas, R.S., J.M. Edmunds, S.C. Marcus, et al., Training and consultation to promote implementation of an empirically supported treatment: a randomized trial. Psychiatr Serv 2012. 63: p. 660-665.

32. Pope, C., Resting evidence: The study of evidence-based medicine as a contemporary social movement. Health 2003. 7: p. 267-282.

33. Choudry, N.K., R.H. Fletcher, and S.B. Soumerai, Systemic review: the relationship between clinical experience and quality of healthcare. Ann Intern Med 2005. 142: p. 260-273.

34. Kazdin, A.E., Evidence-based treatment and practice: new opportunities to bridge clinical research and practice, enhance the knowledge base, and improve patient care. Am Psychol 2008. 63: p. 146-159.

35. Bernal, G. and M.M.D. Rodriguez, eds. Cultural adaptations: tools for evidence-based practice with diverse populations. 2012, American Psychological Association: Washington DC.

36. Roose, S.P., B.R. Rutherford, M.M. Wall, et al., Practising evdeince-based medicine in an era of high placebo response rate: number needed to treat reconsidered. Br J Psychiatry, 2016. 208: p. 416-420.

37. Kazdin, A.E. and M.K. Nock, Delineating mechanisms of change in child and adolescent therapy: methodological issues and research recommendations. J Child Psychol Psychiatry, 2003. 44: p. 1116-1129.

38. Cook, J.M., T. Biyanova, and J.C. Coyne, Barriers to adoption of new treatments: an internet study of practicing community psychotherapists. Adm Policy Ment Health 2009. 36: p. 83-90.

39. Lilienfeld, S.O., L.A. Ritschel, S.J. Lynn, et al., Why many clinical psychologists are resistant to evidence-based practice: Root causes and constructive remedies. Clin Psychol Rev 2013. 33: p. 993-900.

40. Gallo, K.P. and D.H. Barlow, Factors involved in clinician adoption and nonadoption of evidence-based interventions in mental health. Clin Psychol (New York) 2012. 19: p. 93-106.

41. Castonguay, L.G., M.R. Goldfried, S. Wiser, et al., Predicting the effect of cognitive therapy for depression: A study of unique and common factors. J Consult Clin Psychol 1996. 64: p. 497-504.

42. Kendall, P.C., E. Gosch, J.M. Furr, et al., Flexibility within fidelity. J Am Acad Child Adolesc Psychiatry, 2008. 47: p. 987-993.

43. U.S. Department of Health and Human Services, Finding the balance: Program fidelity and adaptation in substance abuse prevention - a state-of-the-art review. 2002, U.S Department of Health and Human Service- Substance Abuse and Mental Health Services Administration Center for Substance Abuse Prevention, www.samhsa.gov: Rockville, MD.

44. Norcross, J.C. and B.E. Wampold, Evidence-based therapy relationships: Research conclusions and clinical practices. Psychotherapy 2011. 48: p. 98-102.

45. Norcross, J.C., ed. Psychotherapy relationships that work (Second edition). 2011, Oxford University Press: New York, NY.

46. Karver, M.S., J.B. Handelsman, S. Fields, et al., Meta-analysis of therapeutic relationship variables in youth and family therapy: The evidence for different relationship variables in the child and adolescent treatment outcome literature. Clin Psychol Rev 2006. 26: p. 50-65.

47. Levitt, H.M., A. Pomerville, and F.I. Surace, A qualitative metaanalysis examining clients' experiences of psychotherapy: a new agenda. Psychol Bull 2016. 142: p. 801-830.
48. Hogue, A., T.J. Ozechowski, M.S. Robbins, et al., Making fidelity an intramural game: localizing quality assurance procedures to promote sustainability of evidence-based practices in usual care. Clin Psychol (New York), 2013. 20: p. 60-77.

49. Court, A.J., A. Cooke, and A. Scrivener, They're NICE and neat, but are they useful? A grounded theory of clinical psychologists' beliefs about and use of NICE guidelines. Clin Psychol Psychother 2016 Nov 25 [Epub ahead of print].

50. Cloitre, M., C. Henn-Haase, J.L. Herman, et al., A multi-site singleblind clinical study to compare the effects of STAIR Narrative Therapy to treatment as usual among women with PTSD in public sector mental health settings: Study protocol for a randomized controlled trial. Trials 2014. 15: p. 197.

51. Karlin, B.E. and G. Cross, From the laboratory to the therapy room: national dissemination and implementation of evidence-based psychotherapies in the U.S. Department of Veterans Affairs health care system. Am Psychol 2014. 69: p. 19-33.

52. Lundahl, B., T. Moleni, B.L. Burke, et al., Motivational interviewing in medical care settings: a systematic review and meta-analysis of randomized controlled trials. Patient Educ Counsel 2013. 93: p. 157-168.

53. VanBuskirk, K.A., Motivational interviewing used in primary care: a systematic review and meta-analysis. J Behav Med 2014;37:768780 .

54. Schaeffer, C.M., E. Bruns, M. Weist, et al., Overcoming challenges to using evidence-based interventions in schools. J Youth Adolesc 2005. 34: p. 15-22.

55. Weisz, J.R. and A.E. Kazdin, eds. Evidence-based psychotherapies for children and adolescents (2nd edition). The Guilford Press: New York, NY.

56. Carr, A., The effectiveness of family therapy and systemic interventions for adult-focused problems. J Fam Ther 2009. 31: p. 46-74.

57. Carr, A., The evidence based for family therapy and systemic interventions for child-focused problems. J Fam Ther 2014. 36: p. 107-157.

58. McHugh, R.K. and D.H. Barlow, Dissemination and implementation of evidence-based psychological interventions: a review of current efforts. Am Psychol 2010. 65: p. 73-84.

59. Najavits, L., Seeking safety: A treatment manual for PTSD and substance abuse. 2002, New York: Guilford Press.

60. Rohde, P., G.N. Clarke, D.E. Mace, et al., An efficacy/effectiveness study of cognitive-behavioral treatment for adolescents with comorbid major depression and conduct disorder. J Am Acad Child Adolesc Psychiatry 2004. 43: p. 660-668.

61. Laska, K.M., A.S. Gurman, and B.E. Wampold, Expanding the lens of evidence-based practice in psychotherapy: a common factors perspective. Psychotherapy, 2014. 51: p. 467-481.

62. Baldwin, S.A. and Z.E. Imel, Therapist effects, in Bergin and Garfield's handbook of psychotherapy and behavior change (6th edition), M.J. Lambert, Editor. 2013: New York, NY. p. 258-297.

63. Aarons, G.A. and A.C. Sawitsky, Organizational culture and climate and mental health provider attitudes toward evidence-based practice. Psychol Serv 2006. 3: p. 61-72.

64. Mukuria, C., J. Brazier, M. Barkham, et al., Cost-effectiveness of an improving access to psychological therapies service. Br J Psychiatry, 2013. 202: p. 220-227.

65. Goodheart, C.D., A.E. Kazdin, and R.J. Sternberg, eds. Evidencebased psychotherapy: Where practice and research meet. 2006, American Psychological Association: Washington DC.

66. Elmore, A., Empirically supported treatments: Precept of percept? Prof Psychol Res Pract 2016. 47: p. 198-205.

67. Whaley, A.L. and K.E. Davis, Culture competence and evidencebased practice in mental health services: a complementary perspective. Am Psychol 2007. 62: p. 563-574.

68. Weisz, J.R., I.N. Sandler, J.A Durlak, et al., Promoting and protecting youth mental health through evidence-based prevention and treatment. Am Psychol 2005. 60: p. 628-648. 